\title{
Diverticular Disease of the Small Bowel
}

\author{
Francisco Emilio Ferreira-Aparicio ${ }^{a}$ Rafael Gutiérrez-Vega ${ }^{b}$ \\ Yolanda Gálvez-Molina ${ }^{c}$ Patricia Ontiveros-Nevares ${ }^{d}$ \\ César Athie-Gútierrez ${ }^{\mathrm{a}}$ Eduardo E. Montalvo-Javéa, b \\ ${ }^{a}$ Servicio de Cirugía General, Facultad de Medicina, Hospital General de \\ México, and ' Departamento de Cirugía, Universidad Nacional Autónoma de \\ México (UNAM), Mexico City; ' Servicio de Patología, Hospital General \\ José G. Parres, and ${ }^{d}$ Servicio de Patología, Hospital del Niño Morelense, \\ Cuernavaca, Mexico
}

\section{Key Words}

Diverticular disease $\cdot$ Intestinal obstruction - Intestinal perforation · Intestinal resection - lleostomy

\begin{abstract}
A diverticulum is a bulging sack in any portion of the gastrointestinal tract. The most common site for the formation of diverticula is the large intestine. Small intestine diverticular disease is much less common than colonic diverticular disease. The most common symptom is non-specific epigastric pain and a bloating sensation. Major complications include diverticulitis, gastrointestinal bleeding, acute perforation, pancreatic or biliary (in the case of duodenal diverticula) disease, intestinal obstruction, intestinal perforation, localized abscess, malabsorption, anemia, volvulus and bacterial overgrowth. We describe the clinical case of a 65-year-old female patient with a diagnosis on hospital admittance of acute appendicitis and a intraoperative finding of diverticular disease of the small intestine, accompanied by complications such as intestinal perforation, bleeding and abdominal sepsis. This was surgically treated with intestinal resection and ileostomy and a subsequent re-intervention comprising perforation of the ileostomy and stomal remodeling. The patient remained hospitalized for approximately 1 month with antibiotics and local surgical wound healing, as well as changes in her diet with food supplements and metabolic control. She showed a favorable clinical evolution and was dismissed from the hospital to her home. We include here a discussion on trends in medical and surgical aspects as well as early handling or appropriate management to reduce the risk of fatal complications.
\end{abstract}

Dr. Eduardo E. Montalvo-Javé, MD, PhD, FACS
Departamento de Cirugía, Facultad de Medicina Circuito Interior, Ciudad Universitaria, Av. Universidad 3000 Delegación Coyoacán, 04510 México, DF (Mexico) E-Mail montalvoeduardo@ hotmail.com 


\section{Introduction}

A diverticulum is a bulging sack in any portion of the gastrointestinal tract. The most common site for the formation of diverticula is the large intestine. The presence of diverticula is called diverticulosis; when these diverticula become inflamed, the disease is termed diverticulitis [1]. The diverticulum forms a pouch in a weakness of the intestine, which generally corresponds to the site at which an artery enters into the muscle layer. It is believed that spasms increase pressure in the intestine, creating more diverticula and enlarging existing ones. Diverticula are classified as real or false: real diverticula are composed of all of the intestine's layers, while false diverticula consist of the uncus of the mucosa and the submucosa [1].

Diverticula are rare prior to the age of 40 years and common after that age. Theoretically, everyone at the age of 90 years has many diverticula. Nearly all persons with diverticula do not appear to have symptoms; however, when the latter present, there can be abdominal cramping of unexplained cause, diarrhea and other disorders of intestinal transit. Stool trapped in the diverticula can cause not only bleeding, but also inflammation and infection, resulting in diverticulitis [2].

The goal of treatment is based on reducing intestinal spasms; consumption of a diet rich in fiber (vegetables, fruits and cereals) is the best solution for reducing these. Diverticulosis does not require surgical intervention. However, giant diverticula do require surgical intervention because they are more likely to become infected and perforated [3]. Males under the age of 50 years with diverticulitis need to be operated on at a rate that is three times higher than in women. When age exceeds 70 years, women are those who require surgery three times more than men [1]. Initial symptoms of diverticulitis include spontaneous abdominal pain, pain during clinical exploration (usually in the lower left part of the abdomen) and fever. X-ray with barium enema to confirm the diagnosis or to study the problem may injure or perforate an inflamed intestine; thus, these tests are usually postponed for a few weeks [2].

Small intestine diverticular disease is much less common than colonic diverticular disease. Its etiology is unknown; it is believed that diverticula develop as a result of abnormalities in intestinal peristalsis, intestinal dyskinesis and very high intraluminal pressure, such as in the colon. The diverticulum emerges only at the site where the mesenteric vessels penetrate into the muscular layer of the small intestine. Unlike Meckel diverticula, the remainder of small intestine diverticula are acquired and the incidence of these is closely related with age $[4,5]$.

Diverticula can be also classified as intra- and extraluminal. Intraluminal and Meckel diverticula are congenital, while extraluminal diverticula can be found at many anatomical sites and are denominated duodenal, jejunal, ileal or jejunoileal [4]. Duodenal diverticula are approximately five times more common than jejunoileal diverticula. The incidence in both types is unknown because these injuries are usually asymptomatic. Diverticula of the small intestine are usually asymptomatic, as in the case of the colon, with the exception of Meckel diverticula [4, 5].

Major complications include diverticulitis, gastrointestinal hemorrhage, gastrointestinal obstruction, acute perforation, pancreatic or biliary (in the case of duodenal diverticula) disease, intestinal obstruction, intestinal perforation, localized 
abscess, malabsorption, anemia, volvulus and bacterial overgrowth. Mortality is influenced by the age of the patient, the nature of the complications and the time that has elapsed before the intervention, and nearly all patients with diverticulitis require hospitalization [6, 7].

There is no predilection by race or gender; however, there is a preponderance of jejunoileal diverticula in males, while duodenal diverticula occur equally in males and females. The majority of duodenal diverticula cases are observed in patients aged $>50$ years, while jejunoileal diverticula are often observed in patients aged $60-70$ years [8].

Physical findings are also associated with the previously mentioned complications. These findings include a sensation of vague or localized pain, rectal bleeding and abdominal fullness. Any sign or symptom is pathognomonic of small intestine diverticular disease; in the absence of complications, medical history and physical examination, findings are often negative and nearly all patients are diagnosed incidentally [9]. The general recommendation is conservative management in asymptomatic diverticular disease. Abdominal pain without evidence of intestinal obstruction or diverticulitis does not require specific treatment; these patients benefit from intake of fiber, bran and cellulose products [10].

We describe a clinical case in which we highlight trends in medical and surgical management as well as early or appropriate management to reduce the risk of fatal complications.

\section{Case Report}

A 65-year-old female patient was admitted to the Emergency Service at night due to 10 days of development characterized by mild to moderately intense pain in the right hemiabdomen, mainly right iliac fossa, which increased in intensity to become a stabbing pain in the right hemiabdomen with radiation to the mesogastrium and hypogastrium, anorexia, nausea, frequent vomiting of food contents, non-quantified fever and weight loss exceeding $10 \mathrm{~kg}$ within 1 month. She had diabetes mellitus and high blood pressure, poorly controlled with antihypertensive medication and an unspecified hypoglycemic medication for 10 years, as well as occasional diarrhea without specific treatment, which had increased in frequency over the past 3 years and was treated by a physician with unspecified antibiotics and antidiarrhetics 3 months prior to her admittance to the hospital.

On arrival, the patient had absent peristalsis, pain on deep palpation in the right iliac fossa with involuntary muscle endurance, and on feeling a mass in the lower right quadrant, data of peritoneal irritation without characteristic appendicular signs. She had a fever of $38.2^{\circ} \mathrm{C}$ and the remainder of vital signs were within normal range. X-ray of the abdomen, standing and supine, with imaging of intestinal obstruction and plastron in the lower right quadrant, and ultrasonography reported an appendicitis-compatible mass in the right flank.

The decision was made to perform surgery, with the following findings: free liquid of gastrointestinal appearance and slightly foul odor, and multiple adhesions in the omentum with intestinal handles and multiple macroscopic intestinal lesions similar to diverticula (fig. 1, fig. 2 , fig. 3 , fig. 4). Laparotomy was performed following trajectory from injuries, which covered the entire small intestine (due to the ligament of Treitz to the ileocecal valve). We also found multiple perforations of the ileocecal valve of nearly $20 \mathrm{~cm}$ and with a total intestinal trajectory of $1 \mathrm{~m}$ from the previously mentioned $20 \mathrm{~cm}$. We carried out an intestinal resection (fig. 5) with Hartmann pouch and right ileostomy. Exploration of the colon was performed without finding edematous or erythematous cecal appendix; we also performed appendectomy, and on exploration the remainder 
of the colon and of the abdomen was found to be without alterations. A Penrose drain was placed in the pelvic cavity and the surgical procedure was completed.

One week after the initial surgical procedure, abdominal distention occurred, with gastrointestinal-type Penrose drain of $1,000 \mathrm{ml}$ in $24 \mathrm{~h}$. The decision was made to conduct emergency exploratory laparotomy surgery due to clinical data indicative of intestinal perforation, with the following findings: intestinal perforation at the stoma and the performance of a $5 \mathrm{~cm}$ resection and remodeling of ileostomy with surgical scrubbing of the cavity with sterile solution. The remainder of the laparotomy was without pathological findings. $48 \mathrm{~h}$ later, the patient was again started on clear liquids, with the ileostomy in good condition and stoma with drainage of $800 \mathrm{ml}$ in $24 \mathrm{~h}$ intestinal-type and Penrose drain with $60 \mathrm{ml}$ drainage in $24 \mathrm{~h}$, with the patient afebrile. Four days after the second procedure, it was decided to initiate with one bottle of intravenous albumin every $24 \mathrm{~h}$ due to hypoalbuminemia of 1 as well as to the presence of septic focus due to Penrose drainage. One week later, we received a preliminary report of small intestine diverticular disease from the Pathology Service. Thus, we decided to obtain new slices to confirm this. Subsequently, the diagnosis of small intestine diverticular disease was confirmed.

During the patient's last week of hospitalization, she evolved favorably with a clean post-surgical wound with granulation tissue present, stoma of ileostomy in good condition with average $24 \mathrm{~h}$ intestinal-type drainage of 350-400 ml, with good tolerance of a semi-solid porridge diet and with the possibility of ambulation and the absence of infection in the perineum. Vital signs were within normal range. We decide to continue the patient's recuperation at home with a weekly appointment at our service each week and daily care of the surgical wound at a health center. The patient continued on a semi-solid diet of porridge during an additional week at home, with the addition of dietary supplements, management with angiotensin-converting enzyme antihypertensive inhibitor and an orally administered hypoglycemic, antacid medicine, and without antibiotics.

\section{Results}

Based on histological slices and microscopic observations by highly qualified personnel, the final diagnosis of small intestine diverticular disease was made (fig, 6). The patient was managed with bowel resections and ileostomy by intestinal perforation, the latter considered among the complications of small intestine diverticular disease. The patient was discharged from the hospital to her home due to improvement and to functional ileostomy, without data of intestinal perforation. The patient is a candidate for remaining with the ileostomy for the rest of her life due to the high risk of bowel perforation and other complications associated with diverticular disease.

\section{Discussion}

This case presented diverticular disease present throughout the small intestine (from the Treitz ligament to the ileocecal valve) and with multiple diverticula. The patient had initiated 10 years previously with frequent diarrhea, which is indicative of a disorder of the peristalsis bowel, such as jejunoileal diverticula. The laboratory reported characteristics of diverticular disease, mainly in leukocytosis and the decrease in hematocrit. In this case, we had only abdominal X-rays and pre-surgical abdominal ultrasonography, because the patient was admitted to the Emergency Service and had been given a presumptive diagnosis of acute appendicitis. An emergency surgical procedure was performed without having had the opportunity of carrying out additional confirmatory studies of diverticular disease. However, the simple abdominal 
X-ray images are consistent with those indicative of the characteristics of diverticular disease, as well as images of intestinal obstruction.

In diverticulitis, nearly all patients require hospitalization; initial intervention includes bed rest, fasting, placement of nasogastric tube, administration of intravenous solutions as well as wide-spectrum antibiotics and evaluation by a specialist. The following were among the main acute complications mentioned: gastrointestinal bleeding, intestinal perforation, intestinal obstruction and intestinal malabsorption [7]. In this case, an exploratory laparotomy was carried out; this is indicated in cases of intestinal perforation, as in this clinical case. Exploratory laparotomy was indicative of the reported case being chronic-evolution diverticular disease with serious complications such as intestinal perforation, bleeding and abdominal sepsis. We must recall that in the majority of cases, diverticular disease is asymptomatic; therefore it goes unnoticed, or in some cases exhibits vague digestive symptoms, such as that of the patient with diarrhea of 10 years. Unfortunately, diagnosis in the best case scenario is often incidental or, as in the majority of cases, until the complications of diverticular disease present, as in the case of our patient. We chose to perform bowel resection and ileostomy due to the high risk of bowel perforation; however, concerning the performance of entero-entero anastomosis, the latter could not be carried out in the first surgical procedure due to the abdominal sepsis present, which is a contraindication for performing entero-entero anastomosis. Due to the lack of metabolic control, associated co-morbidities, and the extent of the disease, the patient is a candidate for remaining permanently with the ileostomy.

\section{Conclusions}

Diverticular disease is usually asymptomatic, detected with the presence of one or more of the several complications described previously, and many cases are diagnosed incidentally during the treatment of these. The clinical case presented is a good example of this situation because, as previously mentioned, the patient had peritoneal irritation data and admittance to the Emergency Service with a pre-operative diagnosis of appendicitis in the elderly. Indicative of diverticular disease, macroscopic lesions were detected during surgery and were subsequently noted by histopathology findings.

During surgery and post-operative management, the procedures were the correct ones and those indicated for intestinal perforation with abdominal sepsis. Small intestine diverticular disease is rare; in this case, it encompassed the entire small intestine. Therefore, there are few options to offer the patient, with ileostomy in perpetuity the most likely procedure to be performed. Diverticular disease is an entity that, as previously mentioned, is diagnosed once the several previously noted complications appear. The key that aided in this case in the survival of the patient and in the improvement of the outcome was effective and timely intervention as well as correct decision-making. 


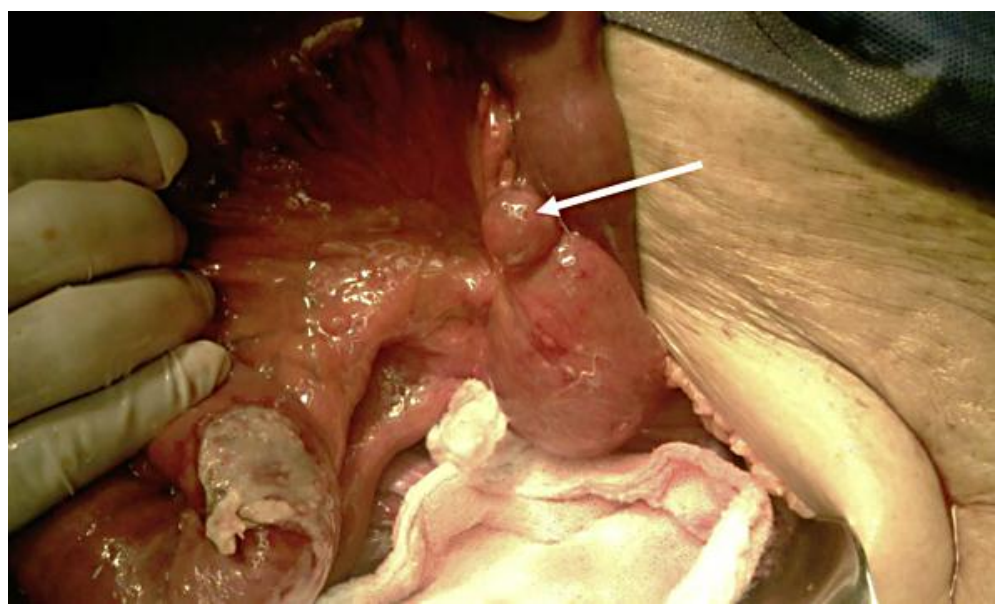

Fig. 1. One diverticulum was found in the mesenteric edge of the jejunum (arrow).

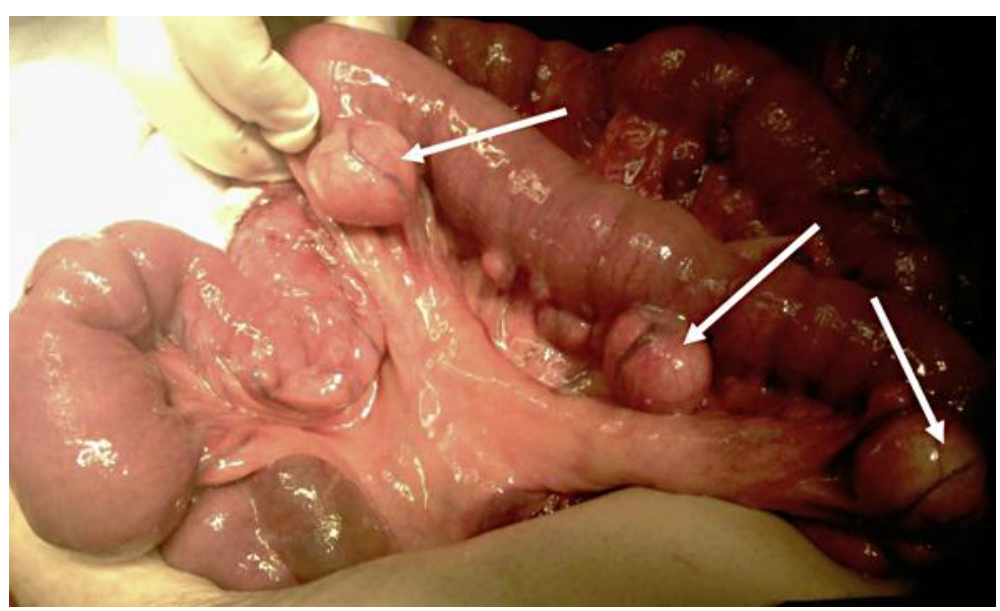

Fig. 2. Multiple diverticula in the mesenteric edge of the jejunum. Three of them are marked with arrows for better identification. 


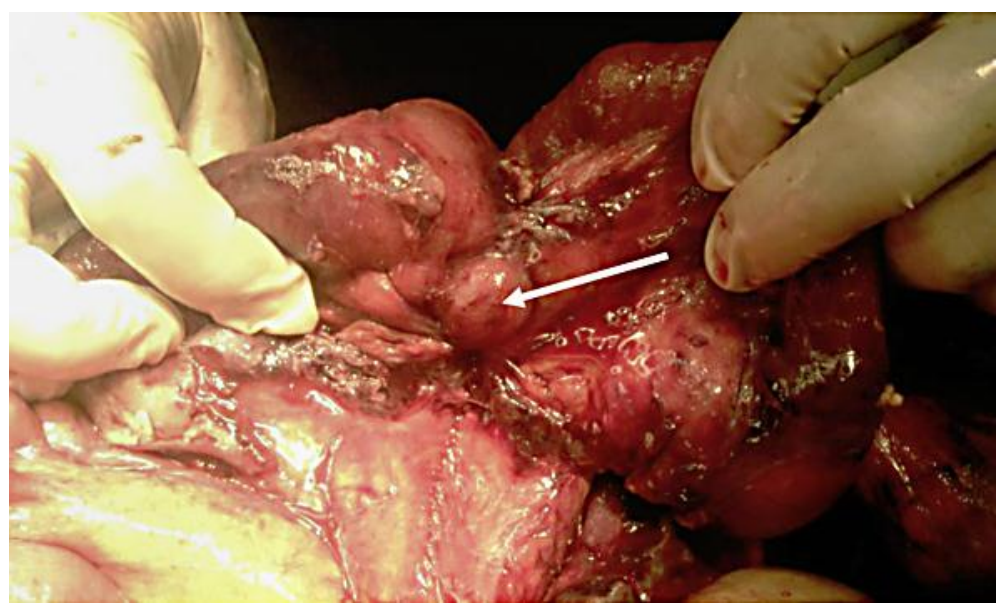

Fig. 3. One diverticulum in the antimesenteric edge of the ileum (arrow).

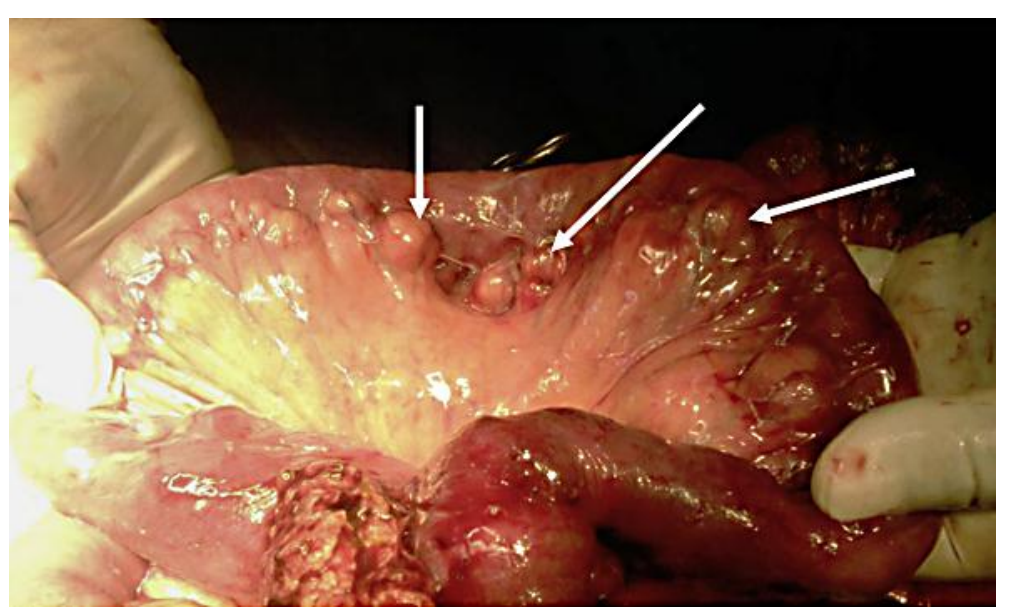

Fig. 4. Multiple diverticula in the mesenteric edge of the ileum (arrows). 


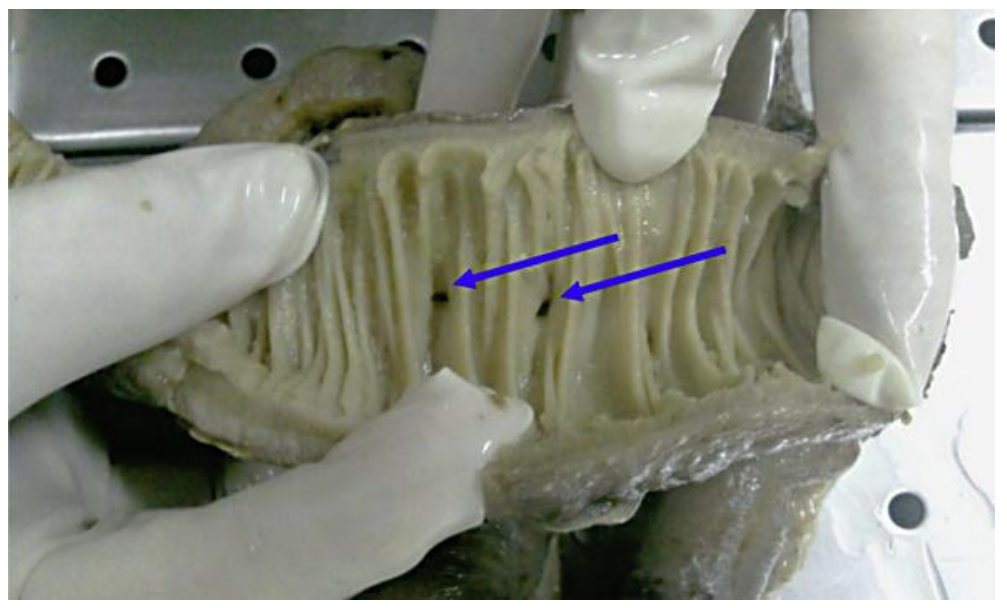

Fig. 5. View of the intestinal lumen of the ileum. Two diverticula can be observed on the antimesenteric edge (arrows).

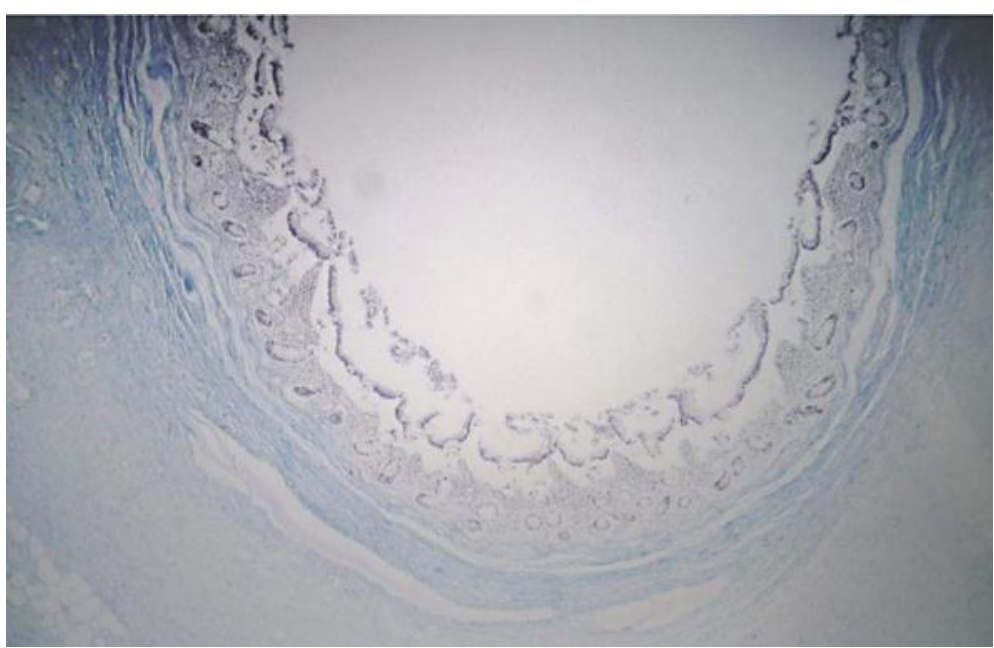

Fig. 6. Histological slice of diverticulum in the ileum (4×, Masson stain). The diverticulum wall as well as all layers that compose it (serosa, muscle, mucous) can be observed.

\section{References}

1 Chow DC, Babain M, Taubin HL: Jejunoileal diverticula. Gastroenterologist 1997;5:78-84.

2 Rubesin SE: Simplified approach to differential diagnosis of small bowel abnormalities. Radiol Clin North Am 2003;41:343-364.

-3 Liu D, Chen L: Management of the total bowel diverticular disease. Hepatogastroenterology 2009;56: 1679-1682.

4 Clarke RC, Ferraro RM, Ozick L, Adediji OS: Diverticulosis, small intestinal. JSP Health Systems Hospital 2010;130:115-118.

5 Albert JG, Lübbert C, Surow A, Zeuzem S: Small bowel diverticula - unknown disease. Z Gastroenterol 2009;47:674-681.

6 Donald JW: Major complications of small bowel diverticula. Ann Surg 1979;190:183-188. 
7 Eckhauser FE, Zelenock GB, Freier DT: Acute complications of jejuno-ileal pseudodiverticulosis: surgical implications and management. Am J Surg 1979;138:320-323.

-8 Akhrass R, Yaffe MB, Fischer C: Small-bowel diverticulosis: perceptions and reality. J Am Coll Surg 1997; 184:383-388.

-9 Wiesner W, Beglinger C, Oertli D, Steinbrich W: Juxtapapillary duodenal diverticula: MDCT findings in 1,010 patients and proposal for a new classification. Belg J Radiol 2009;92:191-194.

10 Colvin HS, Kuenfoo C, Rajab TK, Sayadatas T: Non-surgical management of recurrent perforation of a jejunal diverticulum following previous segmental bowel resection: a case report. J Med Case Rep 2009;3:7318. 